\title{
Active LR Integrator Circuit for Drift-Free Magnetoelastic Transducer
}

\author{
P. GAZDA ${ }^{a, *}$, M. NOWICKI ${ }^{a}$ AND M. KaChNIARZ ${ }^{b}$ \\ ${ }^{a}$ Institute of Metrology and Biomedical Engineering, Warsaw University of Technology, \\ Andrzeja Boboli 8, 02-525 Warsaw, Poland \\ ${ }^{b}$ Industrial Research Institute for Automation and Measurements PIAP, \\ Al. Jerozolimskie 202, 02-486 Warsaw, Poland
}

\begin{abstract}
Current integrator systems usually use active RC integrator circuits. Crucial dificulty associated with this analogue system is the integrator drift. The following paper presents the idea of the active integrator circuit based on inductive and resistive components. This concept allows to eliminate the time drift of the circuit, which is undesired phenomenon resulting from capacitive components working in the traditional negative feedback loop. The SPICE simulations were performed to validate the presented idea. Then, prototype circuit with discrete components was tested. Inductors were based on nanocrystalline and air cores. The developed solution was tested as magnetoelastic sensors transducer, to confirm the ability for long-term, continuous, drift-free, integrator circuit operation. The results were compared with traditional, RC circuit with automatic drift compensation.
\end{abstract}

DOI: 10.12693/APhysPolA.133.745

PACS/topics: 07.55.-w, 75.50.Kj, 75.80.+q

\section{Introduction}

The magnetoelastic effect is based on the phenomenon of changing magnetic properties of the material under the influence of the applied stresses, resulting from minimising internal energy (magnetic, elastic and of anisotropy) in case of change of Crystallographic construction of material caused by these stresses [1]. Magnetoelastic sensors, especial newly developed ones [2], allow to measure compressive stresses, tensile stresses and torque, and are widely utilized in industrial applications [3].

Nowadays, most of integrator circuits, needed to proper operation of magnetoelastic transducer or another sensor based on mathematical integral operation, are using RC principle. Crucial difficulty associated with this is the integrator drift [4]. This effect makes long lasting measurements especially difficult. There are some possibilities to minimize influence of drift, like frequently resetting circuit, or by compensating drift, but it needs additional work and it raises the measurement uncertainty.

It is known that integral operation on constant bias voltage results [5] in linear shift of the output signal. In theory, integration of alternating signal without a DC component has to generate signal with no drift. Nevertheless, due to non-ideality of components (especially difference in base to emitter or gate to source voltage of input transistors) constant offset voltage appears [6]. This phenomenon is called drift and in turn leads to linear increase or decrease of a signal, reaching operational amplifier's saturation level. There are solutions allowing to minimize the drift by periodic or permanent discharg-

*corresponding author; e-mail: p.gazda@mchtr.pw.edu.pl ing the capacitor, although it is complicated and may distort the signal.

\section{General idea}

The presented idea is to replace $\mathrm{RC}[5,7,8]$ integrator circuit with LR one. Equating together equations on current passing through ideal inductance $L$ (with parasitic resistance $R_{L}=0 \Omega$ ) $i_{L}$ and through $i_{R}$ and converting them the following formula is obtained:

$$
V_{\text {out }}=-\frac{R}{L} \int 0^{t} V_{\text {in }}(\tau) \mathrm{d} \tau
$$

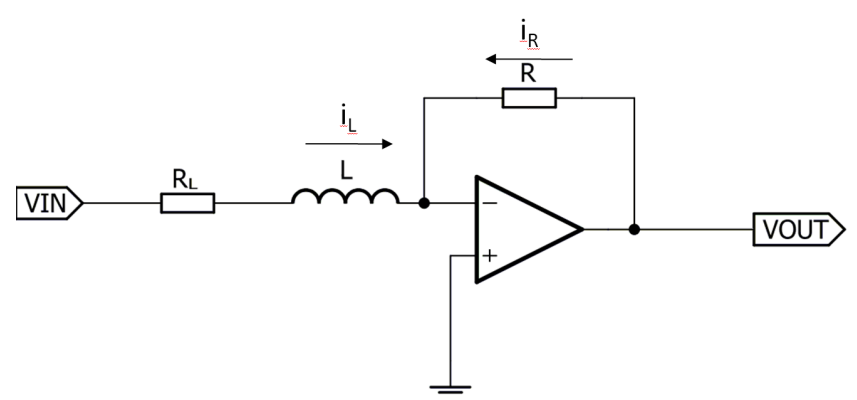

Fig. 1. Electric schematic of the LR integrator circuit.

In fact, beyond the superconductive coil, there is always serial resistance of wire from which the coil is manufactured, which changes character of the circuit to inertial first-order system. Nevertheless, by taking advantage of such a system and choosing appropriate components integration operation may be provided. This occurs for proper frequency range, when impedance of the coil will be considerably higher than the parasitic resistance of the coil. Characteristic parameters of design system like natural frequency $\omega_{0}$, amplification $k_{u}$ and phase shift $\varphi$ are expressed by formulae (2)-(4) [9]: 


$$
\begin{aligned}
& \omega_{0}=\frac{R_{L}}{L}, \\
& k_{u}(\omega)=\frac{V_{\text {out }}}{V_{\text {in }}}=-\frac{Z_{2}}{Z_{1}}=\frac{R}{\omega L+R_{L}}, \\
& \varphi(\omega)=180^{\circ}-\arctan \frac{\omega}{\omega_{0}}=180^{\circ}-\arctan \frac{\omega L}{R_{L}}
\end{aligned}
$$

\section{Simulations}

In magnetoelastic sensors, transducer working frequencies of are of the order $0.1 \mathrm{~Hz} \div 1 \mathrm{kHz}$, depending on the material of the sensor core. It is deemed that signal frequency should be at least 10 times greater than natural frequency of inertial first-order system to ensure correct operation of integration.

Firstly, real standard coil with air core with inductance of $1 \mathrm{H}$ and parasitic resistance of $75.00 \Omega$ was modeled. Simulation was based on [10] National Instrument SPICE program - Multisim. From the parameters of the coil it could be determined that lower working limit will be about $120 \mathrm{~Hz}$ and simulations proved it (Fig. 2). In simulation circuit was tested as a triangle generator - after setting step function on the input of the system, there should be triangle signal on the output.

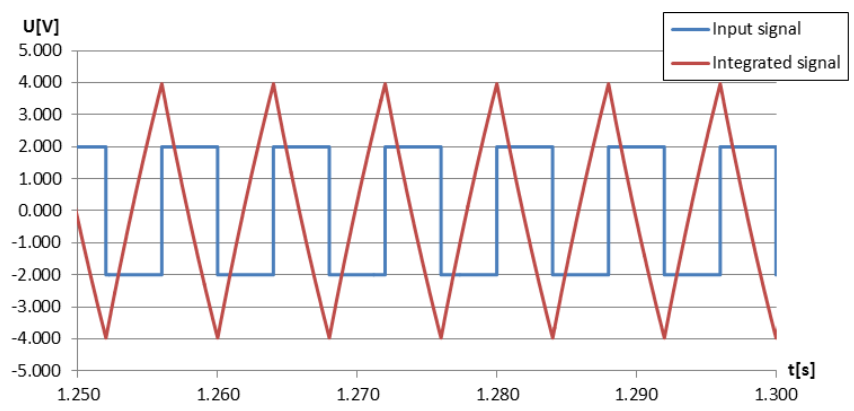

Fig. 2. Output of the simulated system with air core inductor for step function, $f=125 \mathrm{~Hz}[10]$.

Formula (2) implies that, in order to reduce the lower limit of working frequency of the circuit, quotient $R_{L}$ to $L$ should be decreased. Such conditions can be assured mainly through the use of inductors based on core with high permeability, increasing $\mathrm{L}$ while maintaining small $R_{L}$. In order to achieve this, inductor with core made of $\mathrm{Fe}_{73.5} \mathrm{Cu}_{1} \mathrm{Nb}_{3} \mathrm{Si}_{15.5} \mathrm{~B}_{7}$ nanocrystalline alloy was used. Its magnetic permeability is up to 90000 for $10 \mathrm{kHz}$ test frequency.

In contrast to air, which is paramagnetic, used core is ferromagnetic and fundamentally changes simulation and working of such inductors due to phenomena of saturation of the core - coil. Parameters of the hysteresis loop were measured with hysteresisgraph [11]. On the basis of the measurement results, the simplified equation of the hysteresis loop was designed in the form:

$$
B(H)=B_{S} \tanh \left(a \frac{H}{B_{S}}\right) .
$$

That can be entered into simulation software.
$B_{s}$ is a saturation magnetic field density and $a$ is directional factor. Fig. 3 presents operation of the system for step function input for $f=0.7 \mathrm{~Hz}$.

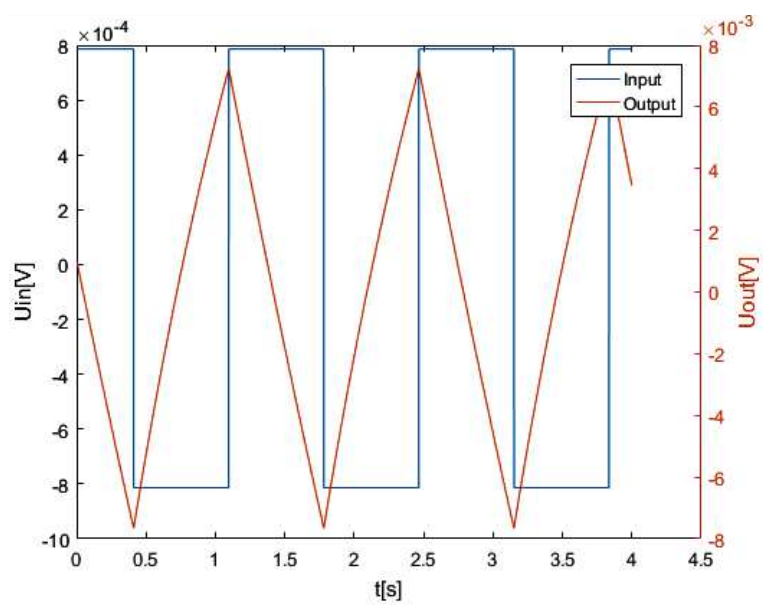

Fig. 3. Output of the simulated system with nanocrystalline core inductor for step function, $f=0.7 \mathrm{~Hz}$.

\section{Experiments}

To test the presented idea, prototype of the device with a replaceable coil was made. Experiments with air core [10] proved proper working of the tested circuit and confirmed that lower limit of working such system is about $120 \mathrm{~Hz}$.

Measurements for nanocrystalline core were started at frequency $1 \mathrm{kHz}$ to test proper working of the system and then decreased to $100 \mathrm{~Hz}, 10 \mathrm{~Hz}$ ending with $0.7 \mathrm{~Hz}$ frequency, that is the lowest frequency, for which the system was operating properly.

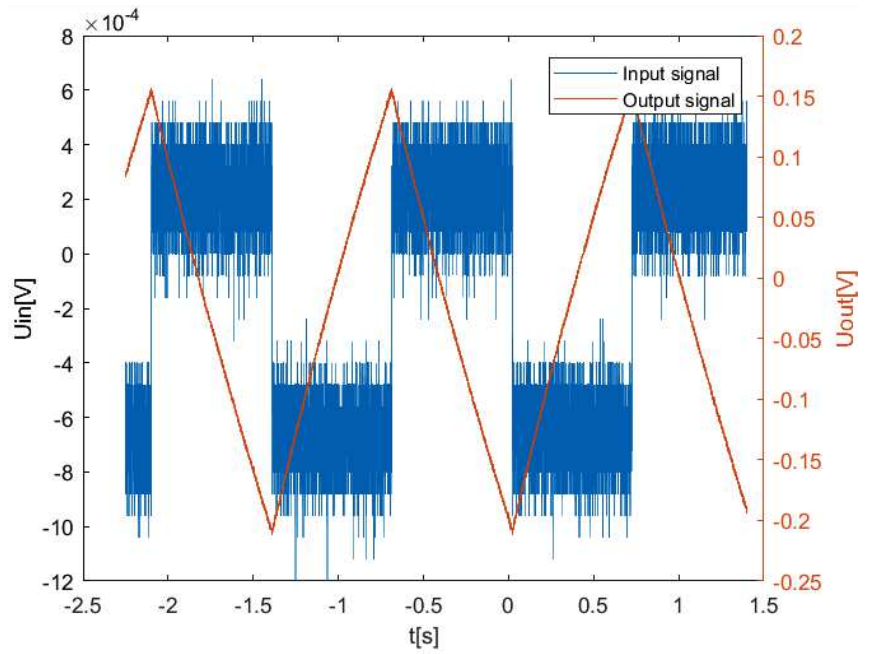

Fig. 4. Acquired signals for the real system with nanocrystalline core inductor for step function, $f=$ $0.7 \mathrm{~Hz}$. 
During the measurements, it was noticed that for decreasing working frequency and decreased impedance of a coil which cased increase in current passing through the coil, it is important to control amplitude of input signal in order not to saturate the coil. Fig. 4 shows acquired signal for $0.7 \mathrm{~Hz}$. System works properly even when the high frequency noise is significant compared to the input signal amplitude.

Appropriate examination of the system operation was to test it as a magnetoelastic transducer. Fig. 5 shows acquired signals for $1 \mathrm{kHz}$ frequency. The results obtained confirm the proper operation of the system and show that working frequency can be much smaller than for the circuit with air core. The transducer will be especially effective for samples with maximum flux density change under stresses influence. Exemplary hysteresis loop for such samples are presented at Fig. 6.

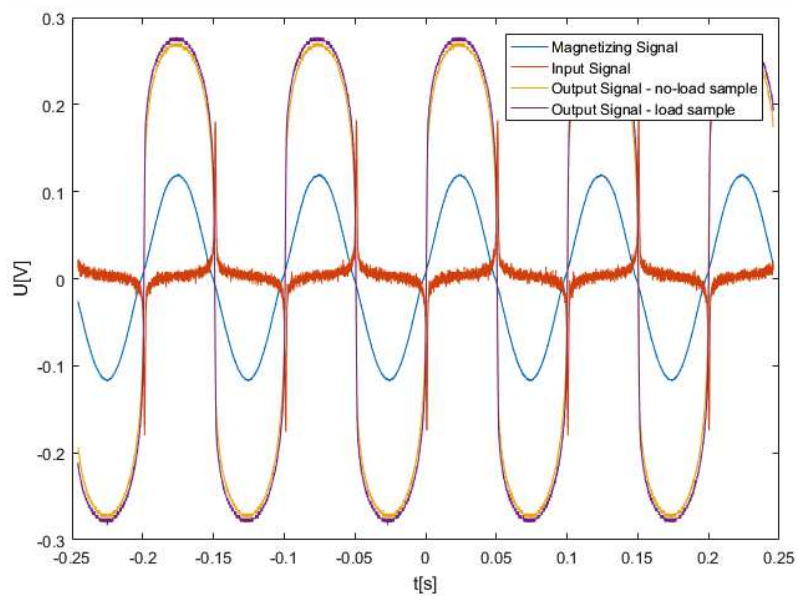

Fig. 5. Output of the real system with nanocrystalline core inductor for signal induced in the magnetoelastic core measurement coil for $f=10 \mathrm{~Hz}$.

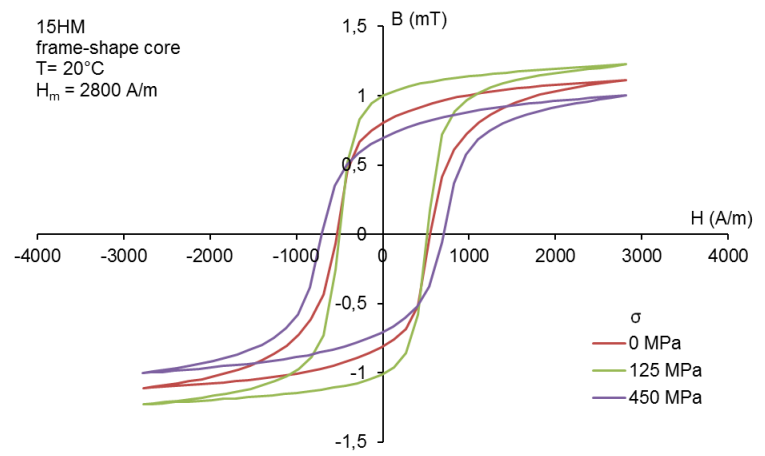

Fig. 6. Magnetic hysteresis loop $B(H) \sigma$ core manufactured from $15 \mathrm{HM}$ steel, with $\mathrm{Hm}=2800 \mathrm{~A} / \mathrm{m}$, for certain values of stretching tension $\sigma=0 \mathrm{MPa}, 125 \mathrm{MPa}$, $450 \mathrm{MPa}[12,13]$.

Measurements for long-term action of circuit were also performed. Commercial fluxmeter shows $10 \mathrm{mV}$ drift per hour. System created by us, for the same conditions had $1 \mathrm{mV}$ drift which can be related to errors in processing of an oscilloscope.

\section{Conclusions}

In presented paper, uncommon kind of integrator circuit was presented. In the practical application, circuit requires a signal conditioning system to prevent saturation of the core. Depending on the changes caused by the applied load, the system alone can be a magnetoelastic transducer or require additional processor systems.

\section{Acknowledgments}

This work was partially supported by the statutory founds of Institute of Metrology and Biomedical Engineering, Warsaw University of Technology (Poland).

\section{References}

[1] W. Fuller Brown Jr., Journal of Applied Physics 36, 994 (1965).

[2] P. Svec, J. Zigo, M. Nowicki, in: Mechatronics Ideas for Industrial Applications, Eds.: J. Awrejcewicz, R. Szewczyk, M. Trojnacki, M. Kaliczyńska, Springer, Cham 2015, p. 381.

[3] A. Bieńkowski, R. Szewczyk, J. Salach, Acta Phys. Pol. A 118, 1008 (2010).

[4] D. Zhang, X. Yan, E. Zhang, S. Pan, Rev. Sci. Instr. 87, 105119 (2016).

[5] H.P. Gavin, R. Morales, K. Reilly, Rev. Sci. Instr. 69 2171 (1998).

[6] P.R. Gray, P.J. Hurst, S.H. Lewis, R.G. Meyer, Analysis and design of analog integrated circuit, Wiley, New York 2009

[7] Z. Pólik, M. Kuczmann, J. Optoelectron. Adv. Mater. 10, 1861 (2008).

[8] E. M. Ga, D. Son, J. G. Bak, S. G. Lee, J. Magn. 8, 160 (2003).

[9] N. Storey, Electronics: a system approach, Pearson Higher Education, Harlow 2013.

[10] P. Gazda, M. Nowicki, M. Kachniarz, M Szudarek, $\mathrm{R}$ Szewczyk, in: Automation 2017 Innovations in Automation Robotics and Measurement Techniques, Eds.: R. Szewczyk, C. Zieliński, M. Kaliczyńska, Springer, Cham 2017, p. 519.

[11] T. Charubin, M. Urbański, M. Nowicki, in: Recent Advances in Systems, Control and Information Technology, Eds.: R. Szewczyk, M. Kaliczyńska, Springer, Cham 2016, p. 593.

[12] M. Kachniarz, D. Jackiewicz, M. Nowicki, A. Bieńkowski, R. Szewczyk, W. Winiarski, in: Mechatronics - Ideas for Industrial Application, Eds.: J. Awrejcewicz, R. Szewczyk, M. Trojnacki, M. Kaliczyńska, Springer, Cham 2016, p. 307.

[13] D. Jackiewicz, R. Szewczyk, J. Salach, in: Mechatronics System and Materials V, Eds.: Z. Gosiewski, Z. Kulesza, Solid State Phenomena, Durnten 2013, p. 466. 